\title{
Flexibility Index of Black-Box Models with Parameter Uncertainty through Derivative-Free Optimization
}

\author{
Fei Zhao ${ }^{1}$, Ignacio Grossmann ${ }^{1}$, Salvador García Muñoz ${ }^{2}$, and Stephen Stamatis ${ }^{2}$ \\ ${ }^{1}$ Carnegie Mellon University \\ ${ }^{2}$ Eli Lilly and Company
}

October 11, 2020

\begin{abstract}
The existing methods of flexibility index are mainly based on mixed-integer linear or nonlinear programming methods, making it difficult to readily deal with complex mathematical models. In this article, a novel solution strategy is proposed for finding a reliable upper bound of the flexibility index where the process model is implemented in a black box that can be directly executed by a commercial simulator, and also avoiding the need for calculating derivatives. Then, the flexibility index problem is formulated as a sequence of univariate derivative-free optimization (DFO) models. An external DFO solver based on trustregion methods can be called to solve this model. Finally, after calculating the critical point of the model parameters, the vertex enumeration method and two gradient approximation methods are proposed to evaluate the impact of process parameters and to evaluate the flexibility index. A reaction model is studied to show the efficiency of the proposed algorithm.
\end{abstract}

\section{Hosted file}

Zhao_DFO paper_1009.pdf available at https://authorea.com/users/366361/articles/486146flexibility-index-of-black-box-models-with-parameter-uncertainty-through-derivativefree-optimization 